\title{
The analgesic effect of combined treatment with intranasal S-ketamine and intranasal midazolam compared with morphine patient-controlled analgesia in spinal surgery patients: a pilot study
}

\author{
This article was published in the following Dove Press journal: \\ Journal of Pain Research \\ 13 February 2015 \\ Number of times this article has been viewed
}

\section{Christine Riediger ${ }^{1, *}$ \\ Manuel Haschke ${ }^{2, *}$ \\ Christoph Bitter ${ }^{3}$ \\ Thomas Fabbro ${ }^{4}$ \\ Stefan Schaeren ${ }^{5}$ \\ Albert Urwyler' \\ Wilhelm Ruppen'}

'Department for Anesthesia, Surgical Intensive Care, Prehospital Emergency Medicine and Pain Therapy, ${ }^{2}$ Clinical Pharmacology, ${ }^{3}$ Hospital Pharmacy, ${ }^{4}$ Clinical Trial Unit, ${ }^{5}$ Orthopedic Department, University Hospital Basel, University of Basel, Basel, Switzerland

*These authors contributed equally to this work
Correspondence: Wilhelm Ruppen Department for Anesthesia, Surgical Intensive Care, Prehospital Emergency Medicine and Pain Therapy, University Hospital Basel, 2I Spitalstrasse,

Basel 403I, Switzerland

$\mathrm{Tel}+4$ I 6I 3286496

Fax +4I 6I 2657320

Email wruppen@gmail.com
Objectives: Ketamine is a well-known analgesic and dose-dependent anesthetic used in emergency and disaster medicine. Recently, a new formulation of S-ketamine, as an intranasal spray, was developed and tested in our institution in healthy volunteers. The authors investigated the effect of intranasal S-ketamine spray combined with midazolam intranasal spray in postoperative spinal surgery patients.

Materials and methods: In this prospective, computer-randomized, double-blinded noninferiority study in spinal surgery patients, the effects of intranasal S-ketamine and midazolam were compared with standard morphine patient-controlled analgesia (PCA). The primary end point was the numeric rating scale pain score 24 hours after surgery.

Results: Twenty-two patients finished this study, eleven in each group. There were similar numeric rating scale scores in the morphine PCA and the S-ketamine-PCA groups at 1, 2, 4, 24,48 , and 72 hours after surgery during rest as well as in motion. There were no differences in the satisfaction scores at any time between the groups. The number of bolus demands and deliveries was not significantly different.

Discussion: In our study, we found that an S-ketamine intranasal spray combined with intranasal midazolam was similar in effectiveness, satisfaction, number of demands/deliveries of S-ketamine and morphine, and number/severity of adverse events compared with standard intravenous PCA with morphine. S-ketamine can be regarded as an effective alternative for a traditional intravenous morphine PCA in the postoperative setting.

Keywords: S-ketamine, intranasal, patient-controlled analgesia, postoperative pain

\section{Introduction}

Ketamine is a well-known analgesic and dose-dependent anesthetic used in emergency and disaster medicine. ${ }^{1}$ Nowadays, ketamine is often used as a very potent analgesic in the perioperative setting. ${ }^{2}$ Several studies have demonstrated the analgesic effectiveness of perioperatively administered ketamine during the acute postoperative period..$^{3,4}$ A systematic review ${ }^{3}$ has shown the analgesic benefit of ketamine, especially in surgery accompanied by high levels of postoperative pain.

There are two optical isomers of ketamine: $\mathrm{S}(+)$ ketamine and $\mathrm{R}(-)$ ketamine. The effects of these drugs are mediated by $N$-methyl-D-aspartate (NMDA), opioids, and muscarinic and different voltage-gated receptors. ${ }^{5}$ Pharmacological studies have shown that S-ketamine is approximately twice as potent as racemic ketamine. ${ }^{6}$ Recently, a new formulation of S-ketamine as an intranasal spray was developed and tested in our 
institution in healthy volunteers. ${ }^{7}$ Intranasally administered S-ketamine was compared with intravenous (IV) and intramuscular S-ketamine. None of the study participants had serious adverse effects or complications after intranasal S-ketamine, and all showed a clear analgesic effect and good absorption of the S-ketamine. The bioavailability of about $70 \%$ was considerably higher than that measured in previous studies $(30 \%-50 \%){ }^{8,9}$ Furthermore, the maximum analgesic effect for the S-ketamine spray was reached after about 3-4 minutes, and thus before the maximum plasma concentration was achieved. ${ }^{7}$ Therefore, S-ketamine spray has several pharmacokinetic and pharmacodynamic advantages compared to morphine. The aim of this pilot study was to investigate the effect of intranasal S-ketamine spray combined with midazolam intranasal spray in a group of postoperative spinal surgery patients compared with traditional patient-controlled analgesia (PCA) with morphine. The rationale for the combination of intranasal S-ketamine and midazolam is the well-known midazolam-antagonizing effect of ketamine-induced psychotomimetic adverse effects. ${ }^{10}$ Furthermore, other studies have reported relaxant and anxiolytic effects of midazolam intranasal spray. ${ }^{11}$ To the best of our knowledge, this is the first clinical study to examine the combination of S-ketamine and midazolam intranasal sprays in adult patients.

\section{Materials and methods General remarks}

This study was registered at http://www.clinicaltrials.gov under the registration number NCT01275547. The study protocol was approved by the local ethics committee (Ethik Kommission beider Basel) and the national regulatory authority (Swiss Agency for Therapeutic Products - Swissmedic). The study was conducted at the Department for Anesthesia, Surgical Intensive Care, Prehospital Emergency Medicine and Pain Therapy, University Hospital of Basel, Switzerland after written informed consent was received from each study patient. The study was performed in accordance with the Declaration of Helsinki and good clinical practice.

\section{Inclusion and exclusion criteria}

Patients scheduled for decompressive surgery for lumbar spinal stenosis, aged $\geq 18$ years, and with a body mass index between 18 and $39.9 \mathrm{~kg} / \mathrm{m}^{2}$ were eligible for our study. Patients with at least one of the following criteria were excluded: unable to give written informed consent; known drug allergies or intolerance to the study medications (morphine, midazolam, ketamine, paracetamol, or metamizol); known allergy to crustaceans or chitosan; patients using snuff on a regular basis; recreational drug abuse; preexisting opioid (tramadol was permitted), ketamine, or midazolam therapy; general physical condition greater than or equal to American Society of Anesthesiologists (ASA) status 4; serious intranasal or epipharyngeal problems; psychiatric disorder; pregnancy; renal failure (clearance $<30 \mathrm{~mL} / \mathrm{min}$ ); and patients with liver failure (Model for End-Stage Liver Disease score $>25$ ).

\section{Study design}

This prospective, computer-randomized, double-blinded, noninferiority study was designed to address pain ratings in a postoperative setting. Patients who met all inclusion criteria were allocated randomly to one of two groups.

The first group was on an alternating S-ketamine intranasal unit-dose spray (6 mg S-ketamine base and $0.5 \mathrm{mcg}$ chitosan-HCL/0.1 mL; unit intranasal dose spray 63607; Aptar [formerly Ing Erich Pfeiffer], Radolfzell, Germany) with midazolam intranasal unit-dose spray $(0.75 \mathrm{mg}$ midazolam base and $0.5 \mathrm{mcg}$ chitosan-HCL/0.1 mL per dose) with a lockout interval of 20 minutes between two applications, and placebo PCA with a lockout interval of 12 minutes with IV saline $0.9 \%$ for 72 hours or until 40 unit-dose sprays were delivered. Chitosan is a tight junction blaster for better resorption.

The second group was on standard PCA with $2 \mathrm{mg}$ morphine IV with a lockout interval of 12 minutes and a placebo intranasal spray (saline $0.9 \%$ and $0.5 \mathrm{mcg}$ chitosan-HCL) with a lockout interval of 20 minutes for 72 hours or until 40 unit-dose sprays were delivered.

The authors of a previous study ${ }^{7}$ used a significantly higher dose of $20 \mathrm{mg}$ of S-ketamine in healthy volunteers. We decided to use a dose of $6 \mathrm{mg}$ of S-ketamine and $0.75 \mathrm{mg}$ of midazolam. The rationale for choosing these significantly lower doses in this study was the pronounced side effects. In order to decrease psychomimetic side effects, in particular, we decided to combine the S-ketamine with midazolam. As the time to maximum concentration in the previous study ${ }^{7}$ was about 21 minutes, we defined a lockout interval of 20 minutes. The primary objective was to determine whether pain on the 0-10 numeric rating scale (NRS) was noninferior, comparing the combined intranasal spray treatment with S-ketamine and midazolam to the standard procedure with morphine PCA after 24 hours. Furthermore, at 1, 2, 4, 24, 48, and 72 hours after surgery, the following secondary outcome parameters were recorded: pain during rest and in motion according to the NRS; total pain relief; amount of demanded and delivered doses of morphine and intranasal S-ketamine/midazolam; 
time until all 40 available sprays per patient were used or premature study termination occurred; amount of rescue medication used during the study period; adverse events; and patient acceptance of the analgesic method and tolerability of the sprays.

A McGill questionnaire ${ }^{12}$ for each study participant was recorded before beginning the study and 72 hours after surgery or after the premature study end. In addition, after the end of the observation period, the 5D-ABZ score ${ }^{13}$ (a five-dimensional questionnaire on extraordinary states of consciousness) was recorded. Four hours and 24 hours after the end of surgery, venous blood samples were collected for analysis of serum concentrations of midazolam and S-ketamine.

After inclusion of the first five patients, we realized the difficulty patients had using the sprays and the PCA correctly, especially immediately after surgery. For this reason, we decided to continuously assist each patient, providing one-on-one care at the bedside during the entire study period using the sprays and the PCA. In doing this, we achieved comparable conditions.

\section{Intra- and postoperative patient management}

Every patient was administered $1.5 \mathrm{mg}$ lorazepam orally about 1 hour before entering the operating room. After arriving at the operating room, the patient was monitored according to the ASA guidelines (blood pressure, $\mathrm{SpO}_{2}$, electrocardiogram) and received an IV line. Anesthesia was induced with propofol (1.5-2 mg/kg body weight) and fentanyl (2-3 $\mu \mathrm{g} / \mathrm{kg}$ body weight). Anesthesia was maintained with propofol (about 6-10 mg/kg body weight per hour) and fentanyl (up to $4 \mu \mathrm{g} / \mathrm{kg}$ body weight per hour), according to the needs of the patient and according to the evaluation of the responsible anesthetist. Ketamine, S-ketamine, clonidine, midazolam, and nonsteroidal anti-inflammatory drugs were forbidden. If the patient's intraoperative anesthetic need was high, sevoflurane could be administered. After surgery, every patient received baseline analgesia with $1 \mathrm{~g}$ paracetamol orally every 6 hours. Rescue medication was IV metamizol $1 \mathrm{~g}$, administered at most, every 6 hours. Baseline analgesia with nonsteroidal anti-inflammatory drugs was not allowed by the orthopedic surgeons for fear of intramedullary bleeding.

In the postanesthesia care unit, patients were monitored according to ASA guidelines (blood pressure, $\mathrm{SpO}_{2}$ ) for about 2-3 hours. At 1, 2, 4, 24, 48, and 72 hours after surgery, the patients' pain (NRS), satisfaction with the analgesic therapy on a scale from 1 (excellent) to 5 (very bad), intranasal spray tolerability, adverse effects (sedation score, nausea, dizziness, pruritus, dry mouth, hypertension, nystagmus, lacrimation, and sialorrhea) and consumption of analgesics were assessed. Furthermore, vital parameters $\left(\mathrm{SpO}_{2}\right.$, blood pressure, and heart rate) were registered. All parameters were recorded in an electronic database.

Each patient was administered 40 unit-dose sprays and received an IV-PCA pump as described earlier. Patients were randomized for either spray verum and IV-PCA placebo or spray placebo and IV-PCA verum. To ensure that patients were not started on midazolam when they requested pain medication at the beginning of the study, the sequence in the verum-spray arm began with an S-ketamine spray with immediate subsequent application of a midazolam spray to alleviate adverse effects of S-ketamine. After the first dual application, the sequence continued with S-ketamine, then midazolam, etc, in alternation.

Each patient had an IV-PCA and a "spray-PCA". The spray-PCA had a lockout interval that was indicated with a blue light. The two PCA systems with different lockout intervals (12 minutes for the morphine IV-PCA, 20 minutes for the spray-PCA) were connected to a single PCA button.

Patients pushed the PCA button each time they were in pain. When the 12-minute lockout interval for the "morphine" pump was over, the patient was given a bolus morphine or saline. Furthermore, when the lamp of the spray-PCA was lit blue, which indicated that the 20-minute lockout interval for the intranasal spray was over, the patient was allowed to receive the next spray. If this analgesic regimen did not satisfy the patient's need, the study was ended and the patient treated with morphine or methadone according to our standard postoperative protocol. If a patient received all 40 unit-dose sprays in less than 72 hours, the study observation period was stopped, and the patient was treated further according to our standard postoperative protocol.

\section{Data and statistical analysis}

Data were collected in an Access 2010 SP3 database, and the analyses were performed with GraphPad Prism (GraphPad version 6.01 for Windows; GraphPad Software, La Jolla, CA, USA). All results are expressed as estimates with $95 \%$ confidence intervals (CIs) unless otherwise stated. As the NRS pain score cannot be expected to be normally distributed, the Wilcoxon rank-sum test was used for estimation together with the method of Bauer ${ }^{14}$ for calculating the two-sided 95\% CI. Spray consumption and plasma concentrations were related by linear regression. 
The difference in the primary end point - NRS pain score 24 hours after surgery (IV morphine PCA minus S-ketamine) - was tested with a Wilcoxon rank-sum test. Two-sided 95\% CIs were calculated according to Bauer. ${ }^{14}$ If a CI lay entirely to the right of the prespecified noninferiority margin $(-2)$, the S-ketamine treatment was considered to be noninferior. Treatment was assigned as indicated by the computer-randomized block list. Patients with at least one end-point measurement were included in the intentionto-treat set.

\section{Results}

In total, 41 patients were screened, 24 of whom could be included between January and May 2012. Two patients had to be excluded before the application of the study medication was started: one patient because of organizational circumstances (surgery late in the evening), and the other patient received midazolam intraoperatively. One patient in the morphine group had to be excluded 30 hours after surgery, because he developed postoperative cognitive dysfunction with delirium. One patient in the ketamine group wished to conclude the study 8 hours after surgery because of insufficient pain relief; this patient also complained about pain levels being too high after changing to a conservative treatment regimen. Table 1 shows the basic data for the 22 patients included who received study medication. All patients underwent decompression; one of them had a decompression and laminectomy. A total of seven consultant surgeons were involved in the operations.

There were similar NRS scores in the morphine-PCA and the S-ketamine-PCA groups at 1, 2, 4, 24, 48, and 72 hours after surgery during rest and in motion (Figures 1 and 2), with a tendency toward lower NRS scores in the S-ketamine intranasal spray group, especially when in motion. The Wilcoxon rank-sum test of the NRS score at 24 hours after surgery did not reveal a significant difference. The $95 \%$ CI for the estimated difference in NRS scores at 24 hours (S-ketamine minus morphine-PCA) was -3 to 2.5 , and thus was not lying

Table I Patient characteristics

\begin{tabular}{lll}
\hline & $\begin{array}{l}\text { Morphine PCA } \\
(\mathbf{n}=\mathrm{II})\end{array}$ & $\begin{array}{l}\text { Ketamine intranasal } \\
(\mathbf{n}=\mathrm{II})\end{array}$ \\
\hline Men & 4 & 5 \\
Women & 7 & 6 \\
Age $($ years $)$ & $76(46-93)$ & $68(38-78)$ \\
Weight $(\mathrm{kg})$ & $75(48-100)$ & $74(66-10 \mathrm{I})$ \\
Height $(\mathrm{cm})$ & $168(155-178)$ & $166(162-185)$ \\
BMI $\left(\mathrm{kg} / \mathrm{m}^{2}\right)$ & $24.7(18.3-33.7)$ & $26.9(23-33.7)$ \\
\hline
\end{tabular}

Note: Values are medians (range).

Abbreviations: PCA, patient-controlled analgesia; BMI, body mass index.

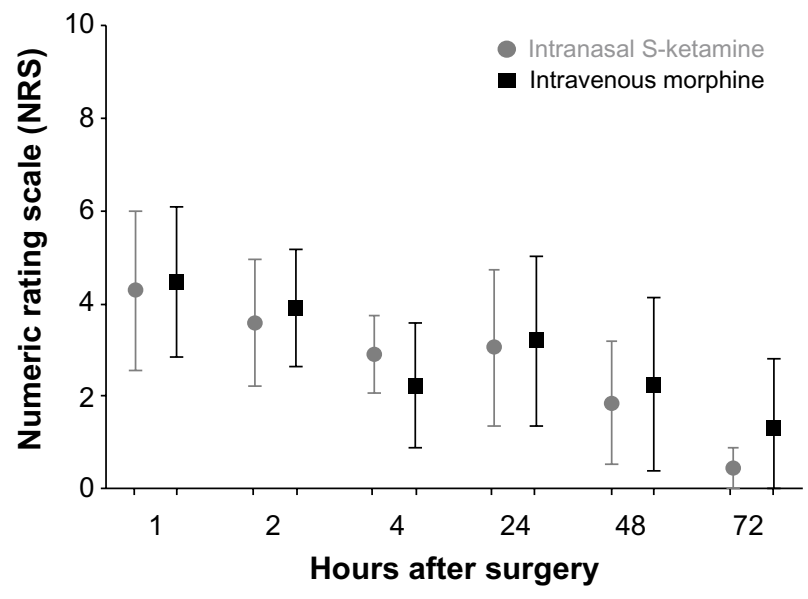

Figure I Distribution of NRS values during rest and I, 2, 4, 24, 48, and 72 hours after surgery in the S-ketamine intranasal spray and the morphine IV-PCA group. Note: Data expressed as means with $95 \%$ Cls.

Abbreviations: IV, intravenous; PCA, patient-controlled analgesia; Cls, confidence intervals.

above (on the right) the noninferiority margin of $\Delta=-2$. Therefore, strictly speaking, we cannot postulate noninferiority of the S-ketamine treatment compared to the morphine treatment for the primary outcome. Assuming a noninferiority margin of $\Delta=3$, all differences lay within the noninferiority margin at all postoperative time points (Figure 3 ).

There was no difference in the satisfaction scores at any time between the groups $(P=0.91)$. The number of bolus demands and deliveries did not differ either $(P=0.96$ for demands, $P=0.81$ for deliveries). Mean use was $30.6(95 \%$ CI 23.3-38.0) in the S-ketamine group and 30.4 (95\% CI 23.3-37.5) in the morphine group. The mean number of demands was $34.9(95 \%$ CI 26.2-43.7) in the S-ketamine and 34.6 (95\% CI 26.6-42.7) in the morphine group (Figure 4).

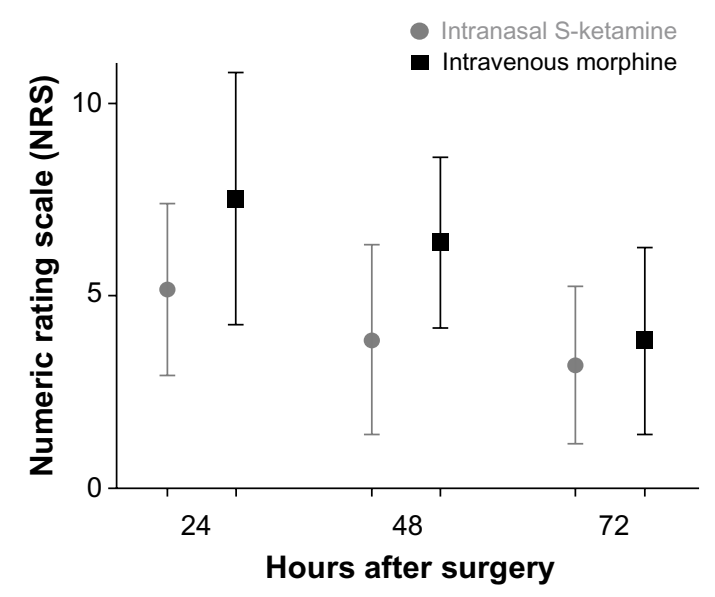

Figure 2 Distribution of NRS values in motion at 24, 48, and 72 hours after surgery in the S-ketamine intranasal spray and the morphine IV-PCA group. Note: Data expressed as means with $95 \% \mathrm{Cls}$.

Abbreviations: IV, intravenous; PCA, patient-controlled analgesia; Cls, confidence intervals. 


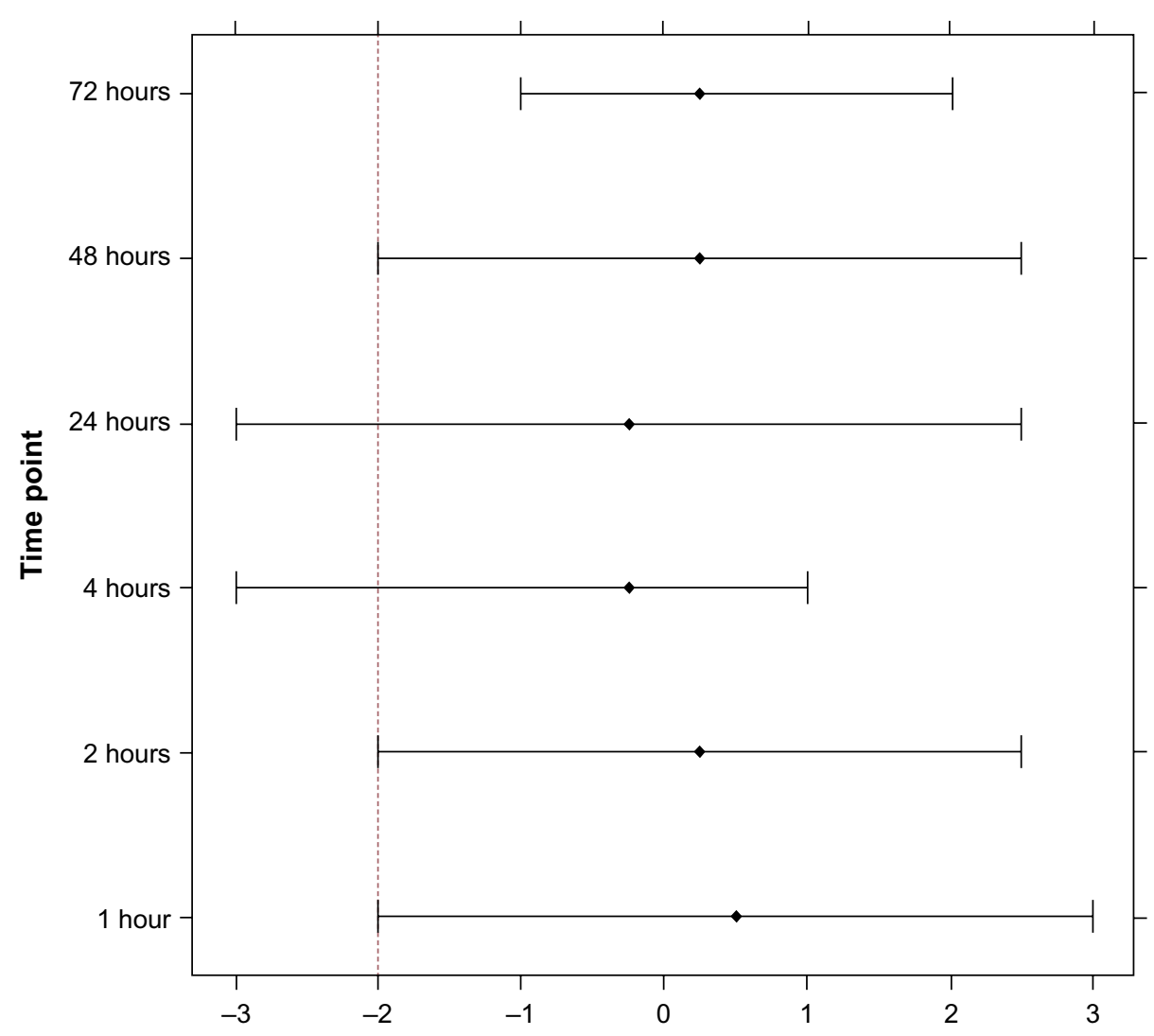

Estimated difference between S-ketamine and morphine PCA

Figure 3 Estimated differences of NRS scores between S-ketamine and morphine treatments, their $95 \%$ Cls, and the prespecified noninferiority margin (dashed line). Abbreviations: PCA, patient-controlled analgesia; Cls, confidence intervals; NRS, numeric rating scale.

Three patients in the ketamine group and three patients in the morphine group were administered all 40 unit-dose sprays after 31, 49, and 68 hours (S-ketamine group) and after 45, 47, and 62 hours (morphine group). Four hours and 24 hours after beginning the study, all patients who received verum nasal sprays had measurable concentrations of midazolam and S-ketamine. Median midazolam and S-ketamine concentrations were $8.7(1.8-12.6) \mathrm{ng} / \mathrm{mL}$ and $16.5(8.5-41.3) \mathrm{ng} / \mathrm{mL}$

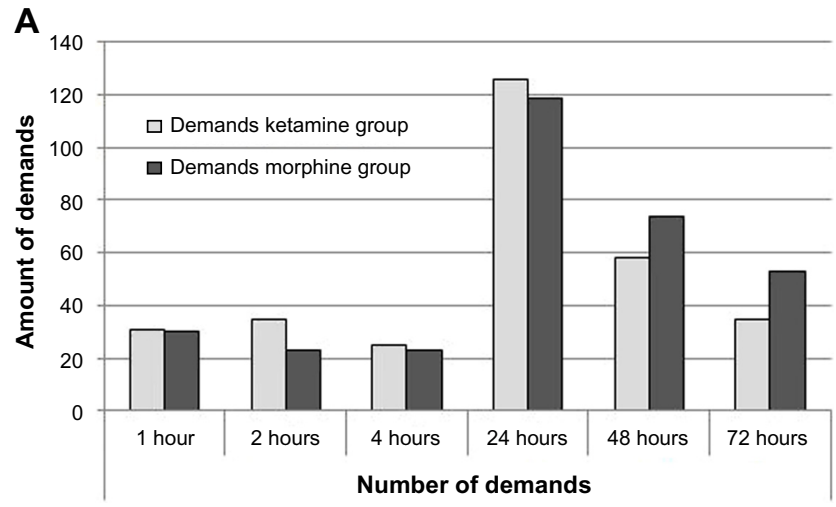

at 4 hours, and $5.3(1.7-16.1) \mathrm{ng} / \mathrm{mL}$ and $11.8(3.2-49.3)$ $\mathrm{ng} / \mathrm{mL}$ at 24 hours, respectively. There was no significant difference in midazolam or S-ketamine concentrations at these two time points (Student's $t$-test, $P>0.5$ for both comparisons).

Plasma concentrations of midazolam at 4 hours correlated with the number of spray applications, $r_{\text {spearman }}=0.74(95 \%$ $\mathrm{CI}_{\text {boot,BCa }}$ 0.20-0.91), while for S-ketamine the correlation

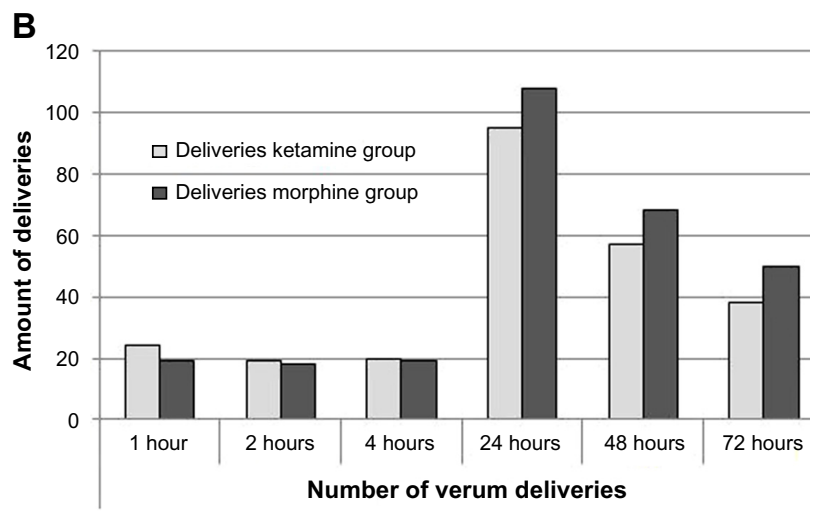

Figure 4 Number of demands (A) and deliveries (B) in the ketamine- and morphine-treatment groups. 
was less clear $r_{\text {spearman }}=0.40,\left(95 \% \mathrm{CI}_{\text {boot,BCa }}-0.42\right.$ to 0.91$)$, due to two patients with relatively low concentrations after four spray applications (Figure 5).

With regard to adverse effects, there were 56 events in the S-ketamine and 59 events in the morphine group (Table 2 ). The total number of adverse effects was not significantly different between the groups, although there was a tendency to a higher frequency of nystagmus in the S-ketamine group and dry mouth in the morphine group. None of the adverse events was serious. The differences between the numbers of chosen words of the five groups of the McGill questionnaire before and after surgery did not differ between the two groups. The consumption of metamizol (rescue medication) was identical in both groups $(P=0.1879$ and $P=0.9719$, respectively).

In the 5D-ABZ score, significantly more auditory alterations were identified in the morphine group $(P=0.0412)$. In the other four main groups, there were no differences ("oceanic boundlessness", $P=0.060$; "anxious ego-dissolution", $P=0.357$; "visionary restructuralization", $P=0.552$; and "vigilance reduction", $P=0.260)$; however, the subgroups "complex hallucinations" $(P<0.0001)$ and "fantasies" $(P<0.0001)$ in the main group "visionary restructuralization" differed in favor of morphine.

\section{Discussion}

In our study, we found no clinically significant differences between a standard IV-PCA with morphine and a new formulation of an intranasal S-ketamine spray combined with intranasal midazolam. Efficacy, satisfaction, demanded and delivered amounts of S-ketamine and morphine, and the number of adverse effects were similar in both groups.

In the proper sense of statistical significance for noninferiority, pain ratings were noninferior after 1, 2, 48, and 72 hours after surgery for a $\triangle \mathrm{NRS} 2$. For a $\triangle \mathrm{NRS} 3$, statistical noninferiority was given for all measured points of time. One could argue that a $\triangle \mathrm{NRS} 2$ is too small to indicate clinical significance, and a $\triangle \mathrm{NRS} 3$ would be more appropriate in an acute pain setting. Furthermore, the NRS distribution in Figures 1 and 2 shows that all NRS scores are distributed in a similar way in both study groups, even with a tendency to lower NRS scores in the S-ketamine group, especially in the groups in motion. In addition, the frequencies of demands for and deliveries of S-ketamine and morphine, satisfaction, and complaints about side effects are similar in both groups. The formal statistical inferiority for a $\triangle \mathrm{NRS}$ 2 at 4 and 24 hours after surgery is a result of relatively large confidence intervals, indicating the small study population of this pilot study.

It is generally accepted that ketamine is an effective analgesic for different postoperative situations..$^{2-4}$ The postoperative use of ketamine reduced opioid consumption, nausea, and vomiting, ${ }^{4}$ and adverse effects were absent or mild. A recent systematic review with meta-analysis ${ }^{3,13}$ showed that the perioperative application of ketamine decreased pain related to very painful surgery, such as major orthopedic, upper abdominal, and thoracic surgery.

We used chitosan as a vehicle that transiently opens tight junctions in the nasal mucous membrane..$^{15,16}$ In the study of Bitter, ${ }^{7}$ the $71 \%$ bioavailability of the ketamine spray with

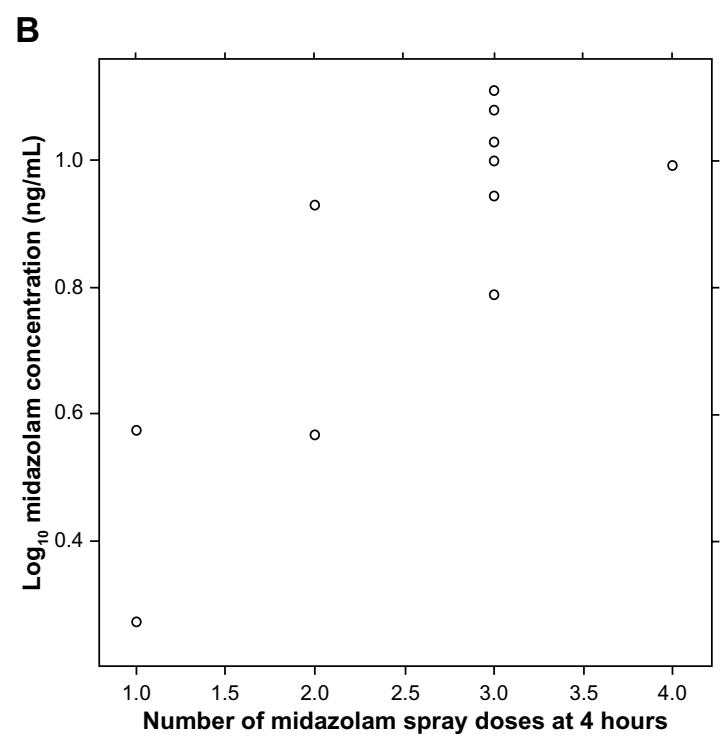

Figure 5 Number of S-ketamine (A) and midazolam (B) unit-spray doses and logarithmic concentrations.

A

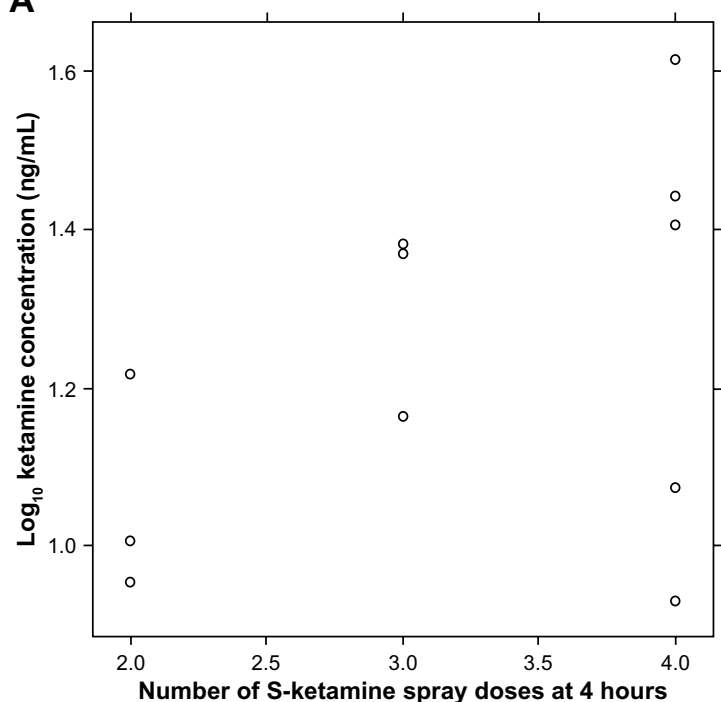

Fire 
Table 2 Reported adverse events

\begin{tabular}{|c|c|c|c|c|c|}
\hline & \multicolumn{2}{|c|}{ Ketamine group } & \multicolumn{2}{|c|}{ Morphine group } & \multirow[t]{2}{*}{$P$-value (patients) } \\
\hline & Patients (n) & Complaints (n) & Patients (n) & Complaints (n) & \\
\hline Nausea & 2 & 3 & 1 & I & 1.0000 \\
\hline Dizziness & 1 & 2 & 0 & 0 & 1.0000 \\
\hline Pruritus & 1 & 1 & 2 & 6 & 1.0000 \\
\hline Dry mouth & 11 & 31 & 9 & 47 & 0.4762 \\
\hline Hypertension & 1 & 1 & 1 & 1 & 1.0000 \\
\hline Sedation & 0 & 0 & 0 & 0 & 1.0000 \\
\hline Nystagmus & 7 & 13 & 2 & 4 & 0.0805 \\
\hline Lacrimation & 3 & 5 & 0 & 0 & 0.2143 \\
\hline Sialorrhea & 0 & 0 & 0 & 0 & 1.0000 \\
\hline Total & & 56 & & 59 & \\
\hline
\end{tabular}

chitosan was considerably higher compared to percentages reported by Christensen et al (33\%), ${ }^{17}$ Yanagihara et al $(45 \%),{ }^{8}$ and Malinovsky et al $(\sim 50 \%) .{ }^{9}$ One of the most important problems in the reported studies ${ }^{9,18-22}$ was the large volume that had to be applied nasally using commercial ketamine products. Volumes of several milliliters far exceed the nasal capacity, especially in pediatric patients who are often included in these studies. It is possible to administer up to $10 \mathrm{mg}$ S-ketamine per $0.1 \mathrm{~mL}$ with single application of our spray. Nevertheless, the nasal application of ketamine is a matter of controversy. ${ }^{23-25}$ As ketamine has a potential risk to be abused, the "needle-free" application with S-ketamine intranasal sprays could promote substance abuse.

PCA systems for postoperative patients should be 1) secure, 2) effective, 3) easy to use, and 4) have a rapid onset. Intranasal sprays can fulfill most of these criteria. The main problem in our study, surprisingly, was the difficulties of patients in using and administering the intranasal spray as well as the IV-PCA. First, the cognitive function of most of the patients was limited immediately postoperatively, complicating their ability to understand the use of the analgesic system (pressing the button of the IV-PCA, applying the intranasal spray, and understanding the meaning of the light used to indicate lockout periods of the spray-PCA), although patients were instructed preoperatively. Second, patients had problems with triggering the spray device. While the sophisticated construction of this device allows for the administration of exactly defined doses, there is considerable resistance when attempting to trigger an application of S-ketamine intranasal spray. This has to be optimized, particularly for commercial forms of the spray. Furthermore, most patients had swollen hands and fingers after lying prone for several hours during the surgery, contributing to the difficulty of using the intranasal spray. In general, due to our experiences in this study, it is possible that we overestimated the abilities of patients to assess and to manage their pain with challenging PCA systems during the postoperative period.

One of the limitations of our study was the limit to just 22 patients. Another limitation was the continuous care of the study patients. On one hand, we do not know if and how one-on-one care influenced the behavior of our patients. On the other hand, one could also argue that the assessment of the patients was more precise.

There are several other potential indications for the use of this S-ketamine intranasal spray in the future. In higher doses, it could also be used in prehospital emergency medicine, especially if IV access is difficult. Furthermore, short interventions in gastroenterology or pneumology or for premedication of children could be performed with intranasal S-ketamine. Finally, S-ketamine intranasal spray could be considered as an alternative, completely noninvasive analgesic procedure in a postoperative outpatient setting. Several studies have reported on the efficacy of ketamine in this setting. ${ }^{26-28}$ As a consequence, the development of a nasal multidose applicator combining S-ketamine and midazolam would be of interest. In addition, the spray could also be used in palliative care situations for patients in severe pain.

In summary, we found S-ketamine intranasal spray combined with intranasal midazolam to be similar in effectiveness, satisfaction, number of demands and deliveries, and number/severity of adverse events compared to standard IV-PCA with morphine.

\section{Acknowledgments}

The authors thank Allison Dwileski, BS (Scientific Secretary, Department for Anesthesia, Surgical Intensive Care, Prehospital Emergency Medicine and Pain Therapy, University Hospital Basel, Basel, Switzerland) for providing editorial assistance. Furthermore, we thank Esther Seeberger for her monitoring work and advice in performing this study. Thanks also to all 
the persons who took one-on-one care of the study patients during the first 72 hours after surgery (in alphabetical order): Katrin Blassmann, Ali-Reza Ghazi-Noori Colucci, Loris D Criscione, Alexander Dotter, Daniela Güdel, Martina Haueter, Hannah Klara Heinrich, Desiree Kaden, David Meier, Rafael Nowak, Diana Reznik, Mirco Schär, Daniela Seeberger, Michael Seeberger, Yasmin Schmid, Tom Siebenaler, Daniel Studer, Veronika Timashkova, Christine Ulmer, Florian Wich, Tanja Wingenbach, Sebastian Wyler, and Melanie Zgraggen. Financial support was provided from institutional and/or departmental sources. WR received a grant from the University of Basel for this study.

\section{Disclosure}

The authors report no conflicts of interest in this work.

\section{References}

1. Svenson JE, Abernathy MK. Ketamine for prehospital use: new look at an old drug. Am J Emerg Med. 2007;25(8):977-980.

2. Himmelseher S, Durieux ME. Ketamine for perioperative pain management. Anesthesiology. 2005;102(1):211-220.

3. Laskowski K, Stirling A, McKay WP, Lim HJ. A systematic review of intravenous ketamine for postoperative analgesia. Can J Anaesth. 2011;58(10):911-923.

4. Bell RF, Dahl JB, Moore RA, Kalso E. Perioperative ketamine for acute postoperative pain. Cochrane Database Syst Rev. 2006;(1): CD004603.

5. Sinner B, Graf BM. Ketamine. Handb Exp Pharmacol. 2008;(182): 313-333.

6. Himmelseher S, PfenningerE. [The clinical use of S-(+)-ketamine-a determination of its place]. Anasthesiol Intensivmed Notfallmed Schmerzther. 1998;33(12):764-770. German.

7. Bitter C. Transmucosal Nasal Drug Delivery: Pharmacokinetics and Pharmacodynamics of Nasally Applied Esketamine [doctoral thesis]. Basel: University of Basel; 2011. Available from: http://edoc. unibas.ch/1310/1/20110314_1408_DissCB_e_version.pdf. Accessed December 16, 2014.

8. Yanagihara Y, Ohtani M, Kariya S, et al. Plasma concentration profiles of ketamine and norketamine after administration of various ketamine preparations to healthy Japanese volunteers. Biopharm Drug Dispos. 2003;24(1):37-43.

9. Malinovsky JM, Servin F, Cozian A, Lepage JY, Pinaud M. Ketamine and norketamine plasma concentrations after iv, nasal and rectal administration in children. Br J Anaesth. 1996;77(2):203-207.

10. Engelhardt W. [Recovery and psychomimetic reactions following S-(+)-ketamine]. Anaesthesist. 1997;46 Suppl 1:S38-S42. German.
11. Koga Y, Sato S, Sodeyama N, et al. Comparison of the relaxant effects of diazepam, flunitrazepam and midazolam on airway smooth muscle. Br J Anaesth. 1992;69(1):65-69.

12. Melzack R. The McGill Pain Questionnaire: major properties and scoring methods. Pain. 1975;1(3):277-299.

13. Dittrich A, Lamparter D, Maurer M. 5D-ABZ: Fragebogen zur Erfassung Außergewöhnlicher Bewusstseinszustände [Questionnaire to assess strange states of consciousness]. 2nd ed. Zurich: PSIN Plus; 2002.

14. Bauer DF. Constructing confidence sets using rank statistics. JAm Stat Assoc. 1972;67(339):687-690.

15. Smith J, Wood E, Dornish M. Effect of chitosan on epithelial cell tight junctions. Pharmac Res. 2004;21(1):43-49.

16. Johnson PH, Quay SC. Advances in nasal drug delivery through tight junction technology. Expert Opin Drug Deliv. 2005;2(2):281-298.

17. Christensen K, Rogers E, Green GA, et al. Safety and efficacy of intranasal ketamine for acute postoperative pain. Acute Pain. 2007;9(4): 183-192.

18. Abrams R, Morrison JE, Villasenor A, Hencmann D, Da Fonseca M, Mueller W. Safety and effectiveness of intranasal administration of sedative medications (ketamine, midazolam, or sufentanil) for urgent brief pediatric dental procedures. Anesth Prog. 1993;40(3):63-66.

19. Louon A, Lithander J, Reddy VG, Gupta A. Sedation with nasal ketamine and midazolam for cryotherapy in retinopathy of prematurity. Br J Ophthalmol. 1993;77(8):529-530.

20. Diaz J. Intranasal ketamine preinduction of paediatric outpatients. Pediatr Anaesth. 1997;7(4):273-278.

21. Weber F, Wulf H, el Saeidi G. Premedication with nasal S-ketamine and midazolam provides good conditions for induction of anesthesia in preschool children. Can J Anaesth. 2003;50(5):470-475.

22. Weber F, Wulf H, Gruber M, Biallas R. S-ketamine and S-norketamine plasma concentrations after nasal and i.v. administration in anesthetized children. Paediatr Anaesth. 2004;14(12):983-988.

23. Bell RF, Kalso E. Is intranasal ketamine an appropriate treatment for chronic non-cancer breakthrough pain? Pain. 2004;108(1-2):1-2.

24. Carr DB, Goudas LC, Denman WT, et al. Safety and efficacy of intranasal ketamine for the treatment of breakthrough pain in patients with chronic pain: a randomized, double-blind, placebo-controlled, crossover study. Pain. 2004;108(1-2):17-27.

25. Lynch ME, Clark AJ. Comment on: Bell RF, Kalso K. Is intranasal ketamine an appropriate treatment for chronic non-cancer breakthrough pain? Pain. 2004;108:1-2. Pain. 2004;110(3):764.

26. Aydin ON, Ugur B, Ozgun S, Eyigör H, Copcu O. Pain prevention with intraoperative ketamine in outpatient children undergoing tonsillectomy or tonsillectomy and adenotomy. J Clin Anesth. 2007;19(2): $115-119$.

27. Erhan OL, Göksu H, Alpay C, Beştaş A. Ketamine in post-tonsillectomy pain. Int J Pediatr Otorhinolaryngol. 2007;71(5):735-739.

28. Conceição MJ, Bruggemann DA Conceição D, Carneiro Leão C. Effect of an intravenous single dose of ketamine on postoperative pain in tonsillectomy patients. Paediatr Anaesth. 2006;16(9):962-967.
Journal of Pain Research

\section{Publish your work in this journal}

The Journal of Pain Research is an international, peer-reviewed, open access, online journal that welcomes laboratory and clinical findings in the fields of pain research and the prevention and management of pain. Original research, reviews, symposium reports, hypothesis formation and commentaries are all considered for publication.

\section{Dovepress}

The manuscript management system is completely online and includes a very quick and fair peer-review system, which is all easy to use. Visit http://www.dovepress.com/testimonials.php to read real quotes from published authors. 